\title{
TAMIZACIÓN DE SALUd VISUAL EN POBLACIÓN INFANTIL: PREVENCIÓN DE LA AMBLIOPÍA
}

Ivonne Andrea Rincón MD*, Nandy Consuelo Rodríguez MD**

\section{Resumen}

Estudio descriptivo en la institución Aldeas Infantiles del barrio Las Cruces de Bogotá DC. (Colombia). Tuvo por objeto describir y confirmar la frecuencia de alteraciones visuales en niños entre seis meses y once años de edad. Se aplicaron pruebas de tamizaje para defectos visuales a 101 niños de estrato socioeconómico uno y dos, según su edad (seis meses a dos años, tres a cuatro años, cinco a seis años y mayores de seis años). Los resultados indican que esta población tiene una prevalencia de defectos visuales similar a la encontrada en otros estudios y que los niños enfermos no tienen la corrección ni el tratamiento apropiados para prevenir defectos mayores en el futuro. Se pretende incentivar la realización de tamizaje en niños por personal médico entrenado para la detección temprana de alteraciones visuales prevenibles.

Palabras clave: ambliopía, niños, tamizaje.

\section{VISION SCREENING IN CHILDHOOD: PREVENTION OF AMBLYOPIA}

\section{Abstract}

This was a descriptive study carried out at the Small Towns for Children of the Las Cruces neighborhood at Bogotá DC. (Colombia). Its purpose was to describe and confirm the frequency of visual impairments in children aged 0.6 months to 11 years. Age-appropriate screening vision tests were applied to 101 boys/girls $(0.6$ months to 2 years, 3 to 4 years, 5 to 6 years and 6 years and older), belonging to socio-economic status one and two. Results revealed that the prevalence of visual impairments in this population is similar to that found in other studies and that these children remain untreated thus failing to prevent greater future consequences. We pretend to boost vision screening in childhood delivered by qualified medical professionals to provide early detection of preventable visual alterations.

Key words: amblyopia, children, screening

\section{Introducción}

Las alteraciones visuales no detectadas en la infancia son frecuentes en preescolares y escolares. La falta de diagnóstico temprano de defectos refractivos y estrabismo hace que se desarrollen afecciones oculares irreversibles después de los diez años de edad. En consecuencia, el déficit de la visión tiene múltiples efectos en todo aspecto de la vida, sobre todo el aprendizaje. Por esta razón es importante que el personal de salud en atención primaria incluyendo enfermeros, optómetras, médicos generales, médicos familiares y oftalmólogos parti-
Fecha recibido: marzo 20 de 2009 - Fecha aceptado: junio 24 de 2009

* Residente III de Medicina Familiar, Fundación Universitaria de Ciencias de la Salud, Hospital de San José, Bogotá DC. Colombia.
** Médica oftalmóloga, Docente Área de Medicina Social y Comunitaria, Directora del Área de Telemedicina, Fundación Universitaria de Ciencias de la Salud, Hospital de San José, Bogotá DC. Colombia. 
cipen en la generación de programas de detección temprana de alteraciones visuales en niños menores de diez años. ${ }^{1}$

Desde el punto de vista estadístico para la OMS por cada millón de personas de la población general 6.000 presentan ceguera de causas prevenibles y de éstos 200 son niños ciegos. Concluye que sólo el $10 \%$ de todas las cegueras son por causas irreversibles. En Colombia no es diferente el panorama y se calculan 7.000 colombianos ciegos por cada millón (296.000 ciegos en todo el territorio). Estas cifras incluyen a toda la población, pero si hablamos de niños el impacto en el aprendizaje y la desadaptación es mayor, observando en Colombia que entre 1 y $2 \%$ de los niños de seis a once años tienen agudeza visual por encima de $20 / 60$ por el mejor ojo y el $75 \%$ corrigen con el agujero estenopeico, lo cual indica que en una gran proporción las causas son defectos de refracción, alteración que puede ser corregida. ${ }^{1}$

Los lugares que reúnen grandes cantidades de infantes como aldeas y jardines infantiles, colegios, comunidades, centros de salud y hospitales, son los ideales para convocar niños y hacer publicidad de demanda inducida y así generar un mayor impacto en la realización de tamizajes preventivos, con el objetivo de reducir cegueras prevenibles en las futuras generaciones de adultos en nuestro país. Son pocos los casos en que un niño mayor de diez años pueda ser tratado de ambliopía, pues a esta edad la maduración de la vía óptica es total. ${ }^{2} \mathrm{El}$ presente artículo describe la realización de una jornada de detección temprana de ambliopía, en una pequeña muestra de niños de seis meses a once años de edad, realizado en una aldea infantil en el suroriente de la ciudad de Bogotá DC.

\section{Ambliopía}

Epidemiología: la frecuencia de ambliopía en la población general mundial es de 1,4 a 4\%. Es la causa más frecuente de pérdida de visión unilateral en niños y adultos jóvenes con consecuencias sociales, laborales y productivas devastadoras. A pesar de ser una patología prevenible o detectable en estadios tempranos, continúa su presentación en diferentes grupos poblacionales. ${ }^{1}$ En Colombia se calcula un $48 \%$ de defectos de refracción en preescola- res (predominando hipermetropía, astigmatismo y miopía), como principal causa de ambliopía en nuestro país. ${ }^{1}$

Definición: los ojos y el cerebro deben trabajar en conjunto para un desarrollo correcto de la visión durante los primeros años de vida. La ambliopía se define como una alteración del desarrollo del procesamiento visual del niño causado por defectos optométricos o de alineación que llevan a una mala percepción visual a nivel del sistema nervioso central que ocurre en la infancia temprana, sin una alteración clara estructural de la vía óptica. Este defecto puede ser uni o bilateral y con diferentes niveles de severidad. ${ }^{3}$

La clasificación más sencilla se realiza según su fisiopatogenia en deprivación visual, desalineación ocular y desenfoque, las tres causas más importantes de ambliopía. La deprivación visual es el defecto que produce una alteración en la percepción de una imagen en la retina, adquirida en etapas iniciales de la vida, es por lo regular unilateral y con frecuencia generada por opacidades de medios como catarata y cicatrices corneanas, o bien malformaciones. La desalineación ocular consiste en la pérdida de fusión de imágenes formadas en las fóveas con dos imágenes generadas en la corteza visual. El cerebro sólo capta la mejor definida o la del ojo dominante. Puede generarse un defecto alternante de la captación de la imagen dando lugar a un desarrollo parcial de la vía óptica bilateral, evitándose así la ambliopía. El desenfoque, última causa descrita de ambliopía, es generado por un defecto refractivo que puede ser aniso o isométrico; el primero en ambos ojos pero con diferencia de dos o más dioptrías entre uno y otro, y el segundo el mismo defecto en ambos ojos que causa la eliminación de los detalles finos de la imagen retiniana, eliminando en la corteza una imagen completa y adecuada. ${ }^{2,4}$

A pesar de que la ambliopía puede generarse en niños sin factores de riesgo o antecedentes importantes, se han descrito algunos que con frecuencia se asocian en los niños con ambliopía: déficit motor, retraso en el desarrollo, prematuridad (menor de 28 semanas de gestación), bajo peso al nacer (menor de $1.500 \mathrm{~g}$ ), antecedente de madre fumadora, consumo de alcohol durante el embarazo y familiares con ambliopía. ${ }^{4}$ Cualquier niño encon- 
trado con alguna de estas alteraciones y además con factores de riesgo, es importante remitirlo a oftalmología para iniciar un manejo apropiado y así prevenir daños irreversibles. ${ }^{4,5}$

\section{Tamizaje}

El tamizaje es una forma de detectar costo-efectivo una enfermedad para evitar progresiones, complicaciones e iniciar el tratamiento oportuno para disminuir consecuencias en la salud de una persona. El de salud visual se debe realizar en todos los niños desde el nacimiento hasta los diez años (aunque entre mayor edad, menor probabilidad de mejoría con tratamiento). ${ }^{5}$ Procederá así:

1. Rojo retiniano: se valora la transparencia de medios observando el reflejo rojo de la retina con el oftalmoscopio en un lugar con poca luz. ${ }^{4}$

2. Detección de estrabismo: cubrir y descubrir cada ojo (cover-uncover): cuando un ojo muestra desplazamiento del reflejo luminoso corneal el explorador tapa el contralateral y si aquel toma la fijación y se desplaza en dirección temporal, el diagnóstico es endotropía. Si se mueve en dirección nasal es una exotropía. ${ }^{4}$

3. Evaluación de agudeza visual: se realiza con la tabla de Snellen a los mayores de tres años, a seis metros de distancia y con valoración monocular, además evaluación de visión cercana. En menores se valora con el comportamiento visual (observando la forma como fija los ojos o reconoce un objeto indicado). ${ }^{4}$ La agudeza visual normal entre cuatro y cinco años debe ser mejor de 20/40, si es peor o hay diferencia de dos líneas entre los dos ojos, presenta ambliopía. Después de los cinco años debe ser mejor de 20/30, si es peor y existe diferencia de dos líneas entre los dos ojos, también es ambliopía. ${ }^{4,2} \mathrm{La}$ Tabla 1 muestra la clasificación de los estadios de agudeza visual según la OMS. ${ }^{2}$

4. Evaluación de estereopsis en niños mayores, según la edad. ${ }^{4}$ de recién nacido hasta dos años ${ }^{2,4}$ (Tabla 2) y mayores de tres años ${ }^{2,4}$ (Tabla 3).

El diagnóstico es de exclusión, pero si es unilateral debe sospecharse cuando presenta diferencia de agudeza visual en los dos ojos (por lo menos de dos líneas). El pronóstico depende de la edad de diagnóstico y tratamiento oportuno, además de la severidad. Incluye la adecuada adherencia al tratamiento y el seguimiento médico. La terapia es individualizada, depende de la causa de la ambliopía y exige un diagnóstico certero. La duración depende del grado de visión que se ha perdido. La base está en obligar al ojo ambliope a trabajar para ver, ocluyendo el ojo sano. Otra forma de tratar es

\begin{tabular}{|c|c|c|}
\hline \multicolumn{3}{|c|}{ Tabla I. Clasificación de los estadios de } \\
agudeza visual según la OMS \\
\hline Grado & $\begin{array}{c}\text { Agudeza visual } \\
\text { corregida en el } \\
\text { mejor ojo }\end{array}$ & Definición \\
\hline 0 & $20 / 20-20 / 60$ & Normal \\
1 & $<20 / 60-20 / 200$ \\
2 & $<20 / 200-20 / 400$ & Déficit visual \\
$3-5$ & $<20 / 400-$ NPL & Déficit visual severo \\
\hline
\end{tabular}

\begin{tabular}{|l|l|l|}
\hline \multicolumn{2}{|c|}{ Tabla 2. Evaluación de estereopsis de recién nacido hasta dos años } \\
\hline Función & \multicolumn{1}{|c|}{ Prueba } & Criterio de referencia \\
\hline Agudeza visual & $\begin{array}{l}\text { Fijación o seguimiento } \\
\text { (comportamiento ocular) }\end{array}$ & Pobre fijación \\
\hline Grandes anormalidades & Examen físico externo & $\begin{array}{l}\text { Observar: ptosis } \\
\text { palpebral, lesiones } \\
\text { corneales o cataratas }\end{array}$ \\
\hline $\begin{array}{l}\text { Alineamiento ocular } \\
\text { Transparencia de medio ocular }\end{array}$ & $\begin{array}{l}\text { Movimiento de los ojos } \\
\text { Leucocoria, leucoma } \\
\text { (opacidad de la córnea) }\end{array}$ \\
\hline
\end{tabular}




\begin{tabular}{|c|c|c|}
\hline Función & Prueba & Criterio de referencia \\
\hline \multirow[t]{2}{*}{ Agudeza visual a distancia } & \multirow[t]{2}{*}{$\begin{array}{l}\text { Tabla de Snellen en letras, núme- } \\
\text { ros o figuras }\end{array}$} & $\begin{array}{l}\text { De } 3 \text { a } 5 \text { años: lectura menor de 20/40 ó diferencia de } \\
\text { dos líneas entre los ojos }\end{array}$ \\
\hline & & $\begin{array}{l}\text { Mayores de } 6 \text { años: lectura menor a 20/30 ó dos líneas } \\
\text { de diferencia entre los ojos }\end{array}$ \\
\hline Grandes anormalidades & Examen físico & $\begin{array}{l}\text { Observar: ptosis palpebral, lesiones corneales o } \\
\text { cataratas }\end{array}$ \\
\hline \multirow[t]{2}{*}{ Alineamiento ocular } & Cover-uncover a 3 metros & Alteración del movimiento de los ojos \\
\hline & Reflejo corneano & Ausencia de reflejo pupilar, asimetría pupilar \\
\hline Transparencia del medio ocular & Rojo retiniano & $\begin{array}{l}\text { Pupila blanca, ausencia de reflejo pupilar, asimetría } \\
\text { pupilar }\end{array}$ \\
\hline
\end{tabular}

haciendo penalización que consiste en aplicar atropina, que genera midriasis, en el ojo sano dos a siete días a la semana, para obligar al ojo ambliope a trabajar bien. Otro recurso es la cirugía, utilizada cuando el daño es estrabismo. ${ }^{3,5}$

\section{Materiales y métodos}

Se realizó un estudio descriptivo transversal en una población de 101 niños de estrato socioeconómico bajo (1 y 2) cuya distribución por edades fue: seis meses a dos años, tres a cuatro años, cinco a seis años y seis a once años. Todos los niños que asisten a Aldeas Infantiles están afiliados a una EPS contributiva o subsidiada. Previo consentimiento de las directivas de las Aldeas Infantiles de Las Cruces (localidad de Bogotá) e información del tamizaje a los padres, se realizaron en 101 niños las siguientes pruebas según su edad:

De seis meses a dos años: (1) comportamiento ocular mediante movimientos oculares guiados por un objeto (juguete) llamativo hacia todas las direcciones; (2) reflejo corneano observando el reflejo de la luz sobre la córnea con el oftalmoscopio; (3) rojo retiniano se realiza con luz de oftalmoscopio dirigiéndola hacia la retina en ambos ojos.

Tres años en adelante: (1) agudeza visual (optotipo de Snellen) realizada a seis metros de la tabla, previa instrucción a cada niño. A los que tienen tres años se les aplica optotipo de elementos que reconocen de cerca; (2) alineamiento ocular, (tapado y no tapado cover/uncover-y reflejo corneal), el primero tapando cada ojo con un oclusor; (3) rojo retiniano mediante la luz de oftalmoscopio directo, dirigiéndola hacia la pupila para que refleje el rojo retiniano. A todos los niños según el rango de edad al que pertenecían se les realizaron las pruebas descritas y se analizó la relación entre género, edad y el número de pacientes con defectos encontrados durante el tamizaje. A los que presentaron alteraciones como defecto de agudeza visual menor de 20/40, alteraciones en el alineamiento o anatómicas grandes, se notificó a los directivos de la Aldea para que fueran enviados a una valoración por su EPS respectiva. Vale aclarar que era requisito estar vinculados con una entidad prestadora de servicios de salud para realizar la valoración por el especialista e iniciar el tratamiento adecuado. Los datos fueron tabulados y procesados por medio del programa ECXEL 2007.

\section{Resultados}

Género: del total (98 niños) de los tamizajes realizados el $47 \%$ (46) son de género femenino y el 53\% (52) masculino (Gráfica 1). Edad: 27\% (27) se encontraron entre seis meses y dos años, $42 \%$ (42) entre tres y cuatro años, $15 \%$ (15) entre cinco y seis años y 14\% (14) mayores de siete años (Gráfica 2). Reflejo pupilar: todos presentaron normalidad en la 


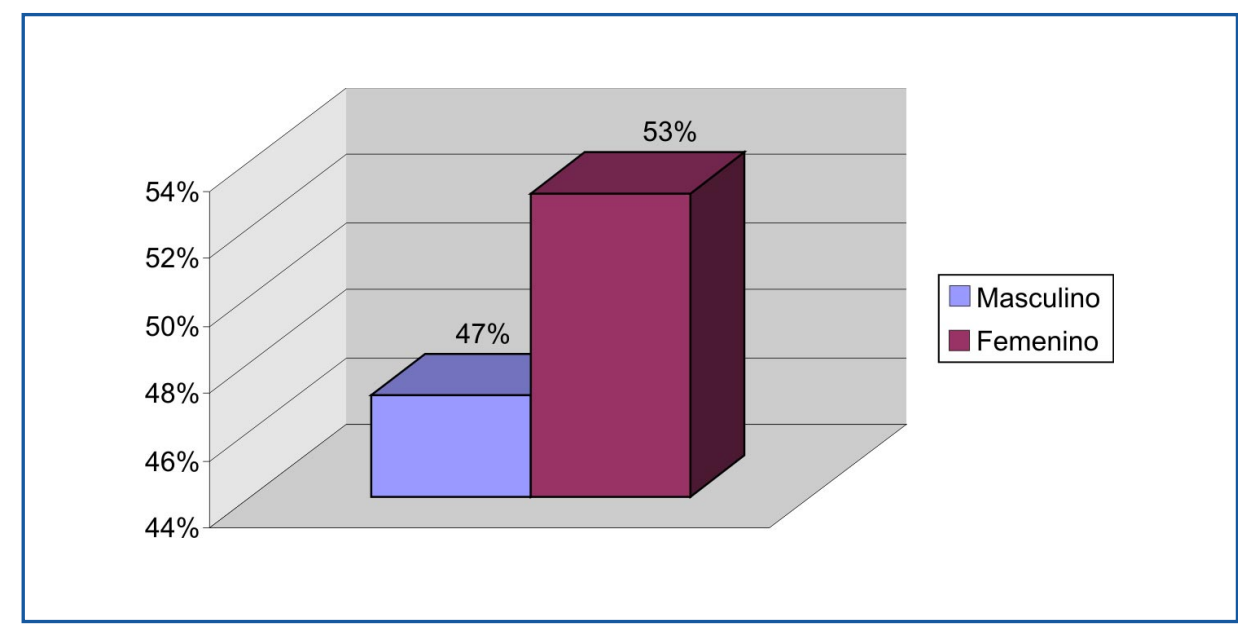

Gráfica I.Distribución por género.

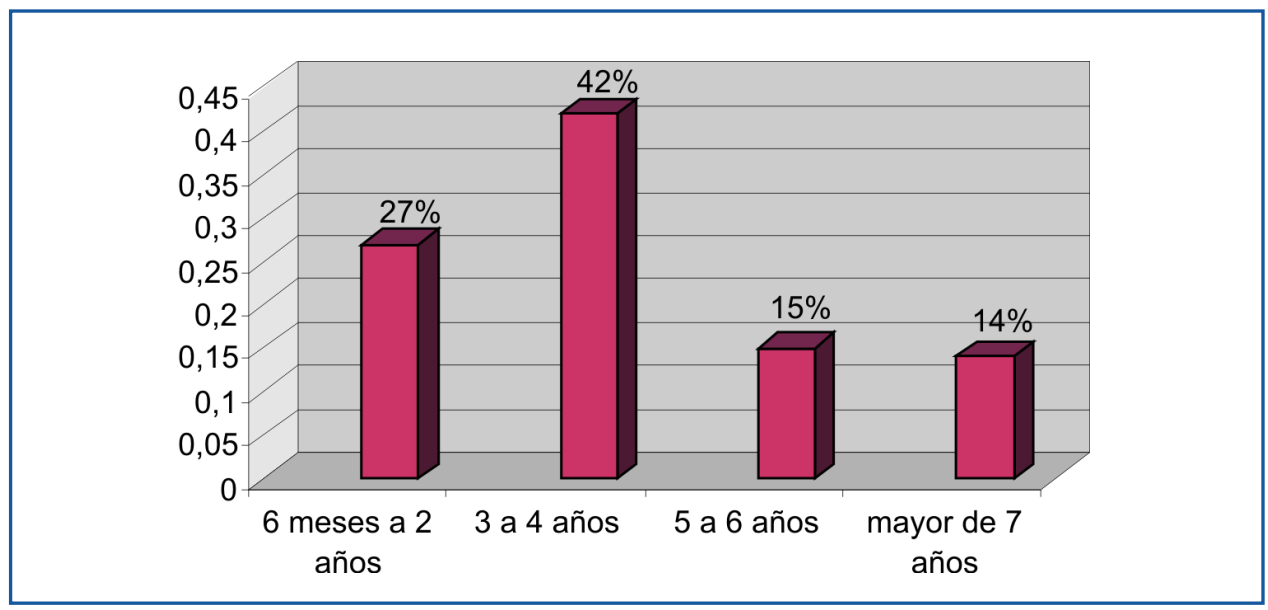

Gráfica 2. Distribución por grupos de edad.

respuesta del reflejo pupilar (Gráfica 3). Rojo retiniano: todos tenían la presencia de rojo retiniano (Gráfica 4). Movimientos oculares: fueron evaluados en todos excepto a tres niños que por su edad fue difícil. Sin embargo, sólo tres presentaron alteraciones dadas por tropías descritas en el examen tapadodestapado (cover-uncover). De los tres niños, dos tienen cuatro años y uno diez años (Gráfica 5). Agudeza visual: se realizó a partir de los tres años de edad; sin embargo en diez no se logró una toma adecuada por falta de comprensión de los optotipos, lo cual se informó a las directivas de las Aldeas Infantiles para que fueran valorados por el especialista. Otros tres niños también fueron notificados por tener inadecuada agudeza visual.
Según la definición de alteración de la agudeza visual de la OMS, 20/50 es normal hasta los cinco años, en el estudio el $16 \%$ de los niños mayores de tres años evaluados tienen agudeza 20/60 o menos. Agudeza visual, ojo derecho: $24 \%$ de niños entre tres a cuatro años presentan agudeza de 20/40. El 9,8\% del total, presentan una alteración mayor o igual a 20/200 (Tabla 4). Agudeza visual, ojo izquierdo: $18 \%$ de los niños entre tres y cuatro años presentan agudeza visual de 20/30. El 9,8\% del total presentan una alteración mayor o igual a 20/ 200 (Tabla 5)

Corrección agujero estenopeico, ojo derecho: sólo el 6,5\% de los mayores de tres años corrigió su agudeza con el agujero estenopeico y la mayoría se encuentra en el rango de mayor o igual a siete años. (Tabla 6). Corrección 


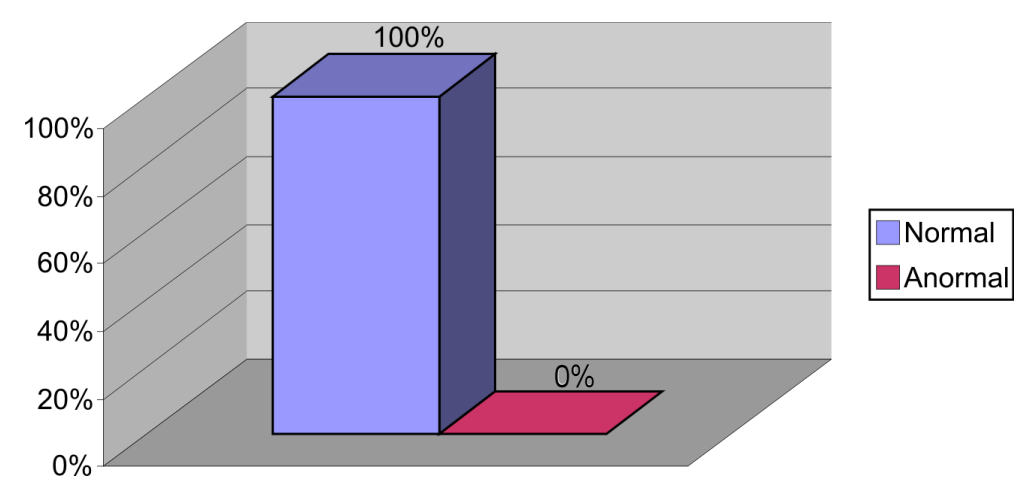

Gráfica 3. Porcentaje de niños con reflejo pupilar.

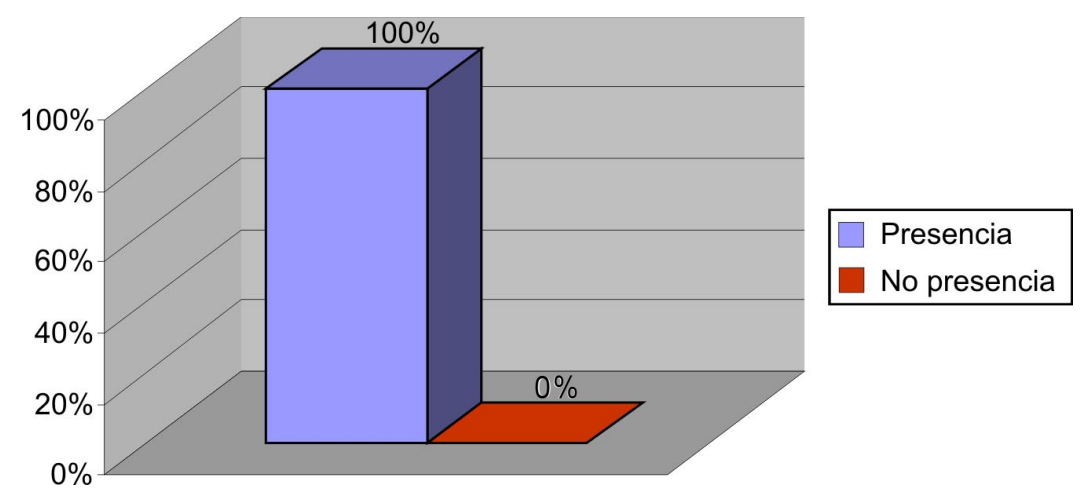

Gráfica 4. Porcentaje de niños con rojo retiniano.

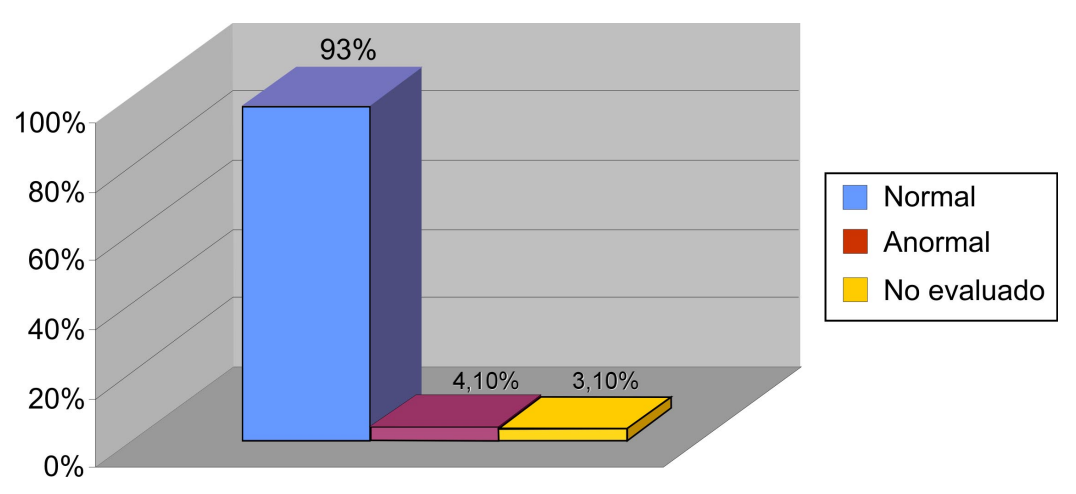

Gráfica 5. Porcentaje de niños con movimientos oculares normales y anormales. 


\begin{tabular}{|c|c|c|c|c|c|c|c|c|}
\hline \multicolumn{1}{|c|}{ Tabla 4. Agudeza visual ojo derecho } \\
\hline Edad/Agudeza & $20 / 20$ & $20 / 30$ & $20 / 40$ & $20 / 50$ & $20 / 60$ & $20 / 70$ & $20 / 200$ & Total \\
\hline $3-4$ años & 0 & 8 & 15 & 2 & 0 & 3 & 4 & 32 \\
$5-6$ años & 4 & 3 & 3 & 3 & 0 & 1 & 1 & 15 \\
$\geq 7$ años & 10 & 1 & 1 & 1 & 0 & 0 & 14 & 6 \\
Total & 14 & 12 & 19 & 6 & 0 & 4 & 6 \\
\hline
\end{tabular}

\begin{tabular}{|c|c|c|c|c|c|c|c|c|}
\hline \multicolumn{10}{|c|}{ Tabla 5. Agudeza visual ojo izquierdo } \\
\hline Edad/Agudeza & $20 / 20$ & $20 / 30$ & $20 / 40$ & $20 / 50$ & $20 / 60$ & $20 / 70$ & $20 / 200$ & Total \\
\hline $3-4$ años & 1 & 11 & 8 & 4 & 0 & 4 & 4 & 32 \\
$5-6$ años & 6 & 1 & 3 & 3 & 0 & 1 & 1 & 15 \\
$\geq 7$ años & 11 & 0 & 1 & 1 & 0 & 0 & 1 & 14 \\
Total & 18 & 12 & 12 & 8 & 0 & 5 & 6 \\
\hline
\end{tabular}

\begin{tabular}{|c|c|c|c|c|c|c|c|c|}
\hline \multicolumn{7}{|c|}{ Tabla 6. Corrección agujero estenopeico ojo derecho } \\
\hline Edad/Agudeza & $20 / 20$ & $20 / 30$ & $20 / 40$ & $20 / 50$ & $20 / 60$ & $20 / 70$ & $20 / 200$ & Total \\
\hline $3-4$ años & 0 & 0 & 0 & 0 & 0 & 0 & 0 & 0 \\
$5-6$ años & 0 & 0 & 0 & 1 & 0 & 0 & 0 & 1 \\
$\geq 7$ años & 2 & 1 & 0 & 0 & 0 & 0 & 0 & 3 \\
Total & 2 & 1 & 0 & 1 & 0 & 0 & 0 \\
\hline
\end{tabular}

agujero estenopeico, ojo izquierdo: sólo el 9,8\% de los niños mayores de tres años corrigió su agudeza con el agujero estenopeico y la mayoría se encuentra en el rango de mayor o igual a siete años (Tabla 7).

Test tapado-destapado (cover-uncover): el 9,8\% de los niños mayores de tres años a los que se les realizó la prueba tiene alteración en la fijación, la mayoría se encuentra entre tres y cuatro años, de éstos $50 \%$ presenta exotropia (Tabla 8).

\section{Conclusiones}

La jornada de tamizaje de salud visual para prevención de ambliopía en Aldeas Infantiles de Las Cruces, realizada durante el mes de junio de 2008, refleja el estado general de la población colombiana.

- En la Aldea existe un mayor porcentaje de niños que de niñas.
- En la población tamizada existe mayor proporción de niños (masculino y femenino) entre tres y cuatro años.

- El 100\% de los niños tamizados no presentaron alteración en rojo retiniano ni en reflejo corneal, lo que indica que no se encontraron otras patologías que generan ambliopía.

- Las alteraciones en los movimientos oculares se ven reflejados en los resultados de la prueba tapadodestapado (cover-uncover) y sólo el 6\% las presentaron.

- De los niños evaluados $16 \%$ mostraron alteraciones en la agudeza visual mayor o igual a 20/60.

- Cerca del 10\% mostraron graves alteraciones en la agudeza visual, una de las principales causas de ambliopía en nuestro medio. 


\begin{tabular}{|c|c|c|c|c|c|c|c|}
\hline \multicolumn{7}{|c|}{ Tabla 7. Corrección agujero estenopeico ojo izquierdo } \\
\hline Edad/Agudeza & $20 / 20$ & $20 / 30$ & $20 / 40$ & $20 / 50$ & $20 / 60$ & $20 / 70$ & $20 / 200$ \\
\hline $3-4$ años & 0 & 0 & 0 & 0 & 0 & 0 & 0 \\
$5-6$ años & 0 & 1 & 0 & 1 & 0 & 0 & 0 \\
$\geq 7$ años & 2 & 2 & 0 & 0 & 0 & 0 & 0 \\
Total & 2 & 3 & 0 & 1 & 0 & 0 & 0 \\
\hline
\end{tabular}

\begin{tabular}{|c|c|c|c|c|c|}
\hline \multicolumn{7}{|c|}{ Tabla 8. Test tapado-destapado (cover-uncover) } \\
\hline Edad $/$ & Exotropía & Endotropía & Exoforia & Endoforia & Total \\
\hline $3-4$ años & 2 & 0 & 1 & 0 & 0 \\
$5-6$ años & 0 & 0 & 0 & 0 & 2 \\
$\geq 7$ años & 1 & 0 & 1 & 1 & 6 \\
\hline Total & 3 & 0 & 2 & & 0 \\
\hline
\end{tabular}

- Un porcentaje muy pequeño corrigió la deficiencia de su agudeza visual con el agujero estenopeico, $4 \%$ para el ojo derecho y $6 \%$ para el izquierdo, diferente al $75 \%$ de los niños que en estudios previos corregía su agudeza visual con el agujero estenopeico, publicados en la revista colombiana Academia de Medicina de Salud Pública.

- Las directivas de las Aldeas Infantiles de Las Cruces fue debidamente informada sobre las alteraciones visuales y se solicitó una atención oportuna por parte de la EPS contributiva o subsidiada a la que los niños tenían derecho.

\section{Referencias}

1. Cuellar Saenz Z. La ceguera: un compromiso de todos. Medicina (Bogotá). 2002; 24(60): 188-96.

2. Colombia. Ministerio de la Protección Social. Norma técnica para la detección temprana de alteraciones visuales y patologías oculares. Bogotá: El Ministerio; 2007.

3. Alañón Fernández FJ, Fernández Pérez J, Ferreiro López S. Oftalmología en atención primaria. Alcalá, España: Formación Alcalá; 2003.

4. Sociedad Colombiana de Oftalmología. Guía para el manejo de ambliopía. Bogotá: La Sociedad; 2005.

5. Nipa Doshi NR, Rodriguez ML. Amblyopia. Am Fam Physician. 2007 Feb 1; 75(3):361-7. 